\title{
NORMING MESHES BY BERNSTEIN-LIKE INEQUALITIES
}

\author{
Marco Vianello
}

Abstract. We show that finite-dimensional univariate function spaces satisfying a Bernstein-like inequality admit norming meshes. In particular, we determine meshes with "optimal" cardinality for trigonometric polynomials on subintervals of the period. As an application we discuss the construction of optimal bivariate polynomial meshes by arc blending.

Mathematics subject classification (2010): 26D05, 42A05, 65T40.

Keywords and phrases: Bernstein-like inequalities, norming meshes, optimal admissible meshes, algebraic polynomials, trigonometric polynomials, subintervals of the period, arc blending.

\section{REFERENCES}

[1] T. Bloom, L. Bos, J.-P. CAlvi And N. Levenberg, Polynomial interpolation and approximation in $\mathbb{C}^{d}$, Ann. Polon. Math., 106, (2012), 53-81.

[2] P. Borwein and T. Erdélyi, Polynomials and Polynomial Inequalities, Springer, New York, 1995.

[3] L. Bos, J.-P. Calvi, N. Levenberg, A. Sommariva and M. Vianello, Geometric Weakly Admissible Meshes, Discrete Least Squares Approximation and Approximate Fekete Points, Math. Comp., 80, (2011), 1601-1621.

[4] L. Bos, S. De Marchi, A. Sommariva and M. Vianello, Computing multivariate Fekete and Leja points by numerical linear algebra, SIAM J. Numer. Anal., 48, (2010), 1984-1999.

[5] L. BOS AND M. ViAnELlo, Low cardinality admissible meshes on quadrangles, triangles and disks, Math. Inequal. Appl., 15, (2012), 229-235.

[6] L. Bos And M. Vianello, Subperiodic trigonometric interpolation and quadrature, Appl. Math. Comput., 218, (2012), 10630-10638.

[7] J. P. CALVI AND N. LeVenberG, Uniform approximation by discrete least squares polynomials, J. Approx. Theory, 152, (2008), 82-100.

[8] G. Da Fies, A. Sommariva And M. Vianello, Algebraic cubature by linear blending of elliptical arcs, Appl. Numer. Math., to appear

[9] G. Da Fies And M. Vianello, On the Lebesgue constant of subperiodic trigonometric interpolation, J. Approx. Theory, 167, (2013), 59-64.

[10] H. Ehlich And K. ZelleR, Schwankung von Polynomen zwischen Gitterpunkten, Math. Z., 86, (1964), 41-44.

[11] K. Jetter, J. StöcKler And J. D. WARD, Norming sets and spherical cubature formulas, in: Advances in computational mathematics (Guangzhou, 1997), 237-244, Lecture Notes in Pure and Appl. Math., 202, Dekker, New York, 1999.

[12] A. KRoó, On optimal polynomial meshes, J. Approx. Theory, 163, (2011), 1107-1124.

[13] F. PiAZZON AND M. Vianello, Analytic transformations of admissible meshes, East J. Approx., 16, (2010), 389-398.

[14] W. PlÉSNIAK, Nearly optimal meshes in subanalytic sets, Numer. Algorithms, 60, (2012), 545-553.

[15] T. J. RivLin AND E. W. CHENEY, A comparison of uniform approximations on an interval and a finite subset thereof, SIAM J. Numer. Anal., 3, (1966), 311-320.

[16] A. Sommariva AND M. VIANELlo, Computing approximate Fekete points by $Q R$ factorizations of Vandermonde matrices, Comput. Math. Appl., 57, (2009), 1324-1336. 